\title{
Validación preliminar del inventario de hipersexualidad en jóvenes
}

\author{
Jesús Castro-Calvo \\ castroj@uji.es \\ RAfAel BALlester-ARnAL \\ rballest@uji.es \\ MARÍA DOLORES GIL-LLARIO \\ dgil@uv.es
}

\section{Resumen}

Introducción: La hipersexualidad es un cuadro clínico que resulta de la combinación de un deseo sexual desmedido y una incapacidad patológica para su control. Se estima que podría afectar en torno al $6 \%$ de la población, si bien la cifra varía dependiendo del instrumento empleado para su identificación. Esta varianza se debe, entre otras razones, al uso de instrumentos no validados en los contextos concretos donde se emplean. Con el objetivo de paliar esta limitación, en este trabajo se presentan los resultados psicométricos preliminares de la adaptación al castellano del inventario de hipersexualidad (de ahora en adelante, IH). Método: Para ello, 600 jóvenes (300 chicos y 300 chicas) de entre 18 y 27 años completaron una versión traducida y adaptada del IH junto con otros instrumentos de evaluación de compulsividad sexual. Resultados: A través de AFE se encontró que el IH comprendía 3 factores que explicaban el $55,5 \%$ de la varianza del cuestionario. Esta estructura factorial se confirmó a través de $\operatorname{AFC}\left(x^{2}=239,4 ;\right.$ G.L. $=145 ; x^{2} /$ G.L. $=1,65 ;$ RMSEA $\left.=, 03[90 \% \mathrm{IC}=, 02-, 04]\right)$. La fiabilidad de la puntuación total y las subescalas osciló entre ,79 y ,91. Así mismo, las correlaciones con escalas afines resultaron positivas y significativas ( $r$ entre $, 47 \mathrm{y}, 71)$. Conclusión: Estos resultados justifican su empleo en la evaluación de la hipersexualidad en jóvenes españoles y su superioridad sobre otros instrumentos utilizados tradicionalmente en la evaluación clínica de esta patología.

Palabras clave: inventario de hipersexualidad, traducción, validación, jóvenes.

\section{Abstract}

Introduction: Hypersexual disorder is a clinical diagnosis characterized by an excessive sexual desire together with a pathological inability to control it. It is estimated that this clinical diagnosis could be affecting about $6 \%$ of the total population, although this figure varies depending on the instrument employed for its diagnosis. One of the most important problems when doing an accurate estimation of its prevalence is related to the use of non-validated assessment instruments. In order to solve this limitation, the 
preliminary results of the Spanish translation of the Hypersexuality Inventory $(\mathrm{IH})$ are presented in this paper. Method: For this purpose, 600 young people (300 boys and 300 girls) between 18 and 27 years old completed a translated and adapted version of the IH together with other instruments to assess sexual compulsivity. Results: Through a factor analysis, we found that the $\mathrm{IH}$ was composed by 3 subscales that explained $55.5 \%$ of the variance. This factorial structure was then confirmed through confirmatory factor analyses $\left(\mathrm{X}^{2}=239.4 ;\right.$ G.L. $=145 ; \mathrm{x}^{2} /$ G.L. $\left.=1.65 ; \mathrm{RMSEA}=.03[90 \% \mathrm{Cl}=.02-.04]\right)$. Reliability for the total score and subscales ranged between .79 and .91 . Moreover, correlations between the $\mathrm{IH}$ and related measures were positive and significant $(r$ between .74 and .71). Conclusion: These results support its employment in the assessment of hipersexuality among Spanish young people and its superiority to other traditional measures to assess this clinical diagnosis.

Keywords: hipersexuality inventory, translation, validation, young people.

\section{Introducción}

La hipersexualidad -también llamada compulsividad sexual o adicción al sexo- es un cuadro clínico que se caracteriza por la presencia de un excesivo deseo sexual (expresado a través de intereses normofílicos) que escapa al control voluntario del paciente que la padece (Kafka, 2010; Winters, Christoff y Gorzalka, 2010). En una propuesta de criterios diagnósticos para su inclusión en el DSM-5, se propuso que un paciente con este cuadro clínico debía cumplir al menos 3 de los 4 criterios siguientes: 1) el tiempo dedicado a la actividad sexual interfiere en la atención a las responsabilidades y obligaciones; 2) el sexo se utiliza como medio para regular estados de ánimo disfóricos (ansiedad, depresión, irritabilidad, etc.) o en respuesta a eventos vitales estresantes (estrés laboral, divorcio, etc.); 3) los intentos por controlar o reducir la actividad sexual no resultan eficaces, y 4) se persiste en la actividad sexual a pesar de los problemas físicos, sociales o emocionales que para el propio paciente o las personas de su entorno supone (Kafka, 2010). Algunas investigaciones han demostrado además que en la hipersexualidad están también presentes síntomas clásicos de cualquier adicción a una sustancia tóxica como la tolerancia (la persona cada vez requiere de actividades sexuales más intensas o de mayor duración para alcanzar la satisfacción sexual del inicio) o la abstinencia (la persona padecería síntomas que en la adicción al sexo tomarían la forma de problemas emocionales -irritabilidad, abatimiento, ansiedad, etc. - tras periodos sin poder saciar su impulso sexual) (Wines, 1997).

Algunos estudios sostienen que la prevalencia de esta patología se situaría en torno al $6 \%$ (Hagedorn, 2009), si bien las estimaciones varían dependiendo del criterio para su identificación. A partir de cuestionarios y escalas de screening, la prevalencia estimada se situaría entre el 3-17,4 \% en hombres y el 1,2-32,2 \% en mujeres (Rettenberger, Klein y Briken, 2015), rango que refleja la enorme variablidad entre escalas a la hora de identificar este cuadro clínico. En una revisión de los principales instrumentos de medida para la evaluación de este cuadro clínico, Womack, Hook, Ramos, Davis y Penberthy (2013) advierten de algunos problemas que comprometen la fiabilidad de estas escalas. Entre ellos, los más importantes se derivan del uso de instrumentos sin validar o validados únicamente en su formato original $-y$ no en cada una de las versiones y traducciones empleadas-.

Con el objetivo de evaluar la convergencia diagnóstica entre instrumentos de screening, Castro-Calvo, Ballester-Arnal y Gil-Llario (2015) realizaron un estudio en el que administraron 
las tres principales escalas de evaluación de la hipersexualidad (el inventario de hipersexualidad $\mathrm{IH}$, la escala SCS de compulsividad sexual y el cuestionario SAST de adicción al sexo) a un grupo de 600 jóvenes españoles (300 chicas y 300 chicos). Lo que encontraron fue que, mientras que la correlación entre escalas era alta ( $r$ entre, 529 y ,732), la convergencia diagnóstica era tan solo del $46,3 \%$ en el mejor de los casos. Una de las principales limitaciones de este trabajo era que tan solo una de las tres escalas empleadas había sido validada previamente en el contexto español, de modo que, con el objetivo de paliar las limitaciones derivadas del uso de instrumentos que no han demostrado su utilidad clínica a través de un procedimiento de validación, se procedió a realizar una validación preliminar de otro de los cuestionarios empleados en dicho estudio: el SAST (Castro-Calvo, Ballester-Arnal, Salmerón-Sánchez y GilLlario, 2016). Reconociendo nuevamente la importancia de la validación de cualquier instrumento empleado en la evaluación de este cuadro clínico, en este trabajo se presentan los resultados psicométricos de la adaptación al castellano de una de las escalas de screening más utilizadas para la identificación de la hipersexualidad en el ámbito clínico y empírico: el inventario de hipersexualidad (Reid, Garos y Carpenter, 2011).

\section{Método}

\section{Participantes}

En este estudio participaron un total de 600 jóvenes de Castellón de la Plana (España) de entre 18 y 27 años ( $x=20,67$; DT = 2,22). De ellos, 300 eran mujeres (50 \%) y los 300 restantes hombres. Respecto a la orientación sexual, una amplia mayoría se declaraba heterosexual $(91,5 \%)$, seguido de homosexuales (5\%) y finalmente bisexuales $(3,2 \%)$. La mayoría afirmaron ser ateos $(58,7 \%)$ o creyentes no practicantes (35,8\%). La distribución de porcentajes de participantes con y sin pareja estable fue prácticamente equivalente (51 \% y $49 \%$ respectivamente).

Para el análisis de la validez convergente del instrumento se tomó un subconjunto de la muestra formado por 200 participantes (100 hombres con una edad media de 20,83 años y 100 mujeres con una edad media de 20,50 años) a los que se les administró dos cuestionarios de evaluación complementarios.

\section{Instrumentos}

Aparte de un cuestionario ad hoc de datos demográficos (sexo, edad, nacionalidad, creencias religiosas, etc.), todos los participantes completaron las siguiente batería:

Inventario de hipersexualidad ( $\mathrm{IH}$, Reid y cols., 2011): este instrumento de 19 ítems con formato de respuesta tipo Likert $(1=$ nunca $/ 5=$ muchas veces $)$ se diseñó para evaluar los principales síntomas de hipersexualidad. En población clínica, la estructura factorial consta de tres escalas: afrontamiento, control y consecuencias. Además, el cuestionario permite la obtención de una puntuación total que oscila entre 19 y 95. Los autores de la versión original obtuvieron una fiabilidad de entre 0,89 y 0,95 para las diferentes escalas.

Cuestionario de adicción al sexo de Carnes (1983) en su versión validada en castellano (SAST, Castro-Calvo y cols., 2016): cuestionario de 25 ítems con un formato de respuesta dicotómico (sí/no) que permite la obtención de una puntuación entre 0 (sin síntomas de adicción al sexo) y 25 (cuadro clínico severo). En la versión traducida y validada al castellano, los autores hallaron una estructura factorial de 4 factores (falta de control de impulsos sexuales, síntomas de adicción al sexo, interferencia familiar y ocultación de la actividad sexual) que 
explicaba el 38,38 \% de la varianza del cuestionario. La fiabilidad de la escala general y las subescalas osciló entre, 51 y, 81 .

Escala de compulsividad sexual de Kalichman y Rompa (1995)

en su versión validada en castellano (scs, Ballester-Arnal, Gómez-Martínez, Gil-Llario y Salmerón-Sánchez, 2013): escala de 10 ítems con formato tipo Likert (4 opciones de respuesta que van de «nada característico de mí» a «muy característico de mí») empleada para estimar el grado de control sobre la propia conducta sexual. En una muestra equivalente a la empleada en este estudio, los autores obtuvieron una solución factorial de dos dimensiones: una dimensión de interferencia de la conducta sexual y otra de control de impulsos sexuales. La fiabilidad y estabilidad temporal fue de 0,83 y 0,72 .

El subgrupo de la muestra empleado para el cálculo de la validez convergente completó una batería con dos medidas adicionales:

Escala de búsqueda de sensaciones sexuales de Kalichman y Rompa (1995): escala de 11 ítems con el mismo formato de respuesta idéntico que el scs que evalúa la tendencia a experimentar prácticas sexuales intensas y novedosas -independientemente de su riesgo-. De ahí que algunos estudios encuentren relación con ciertas prácticas sexuales de riesgo (Kalichman y Rompa, 1995).

Cuestionario de adicción al cibersexo de Delmonico (1997) en su versión validada en castellano (Ballester-Arnal, Gil-Llario, Gómez-Martínez y Gil-Julià, 2010): compuesto por 25 ítems de respuesta dicotómica (verdadero/falso), este instrumento evalúa el grado en que la conducta sexual online es o no problemática. La suma de sus ítems permite la obtención de un índice general de adicción al cibersexo y puntuaciones en cinco escalas: 1) compulsividad sexual online (síntomas de consumo patológico de cibersexo), 2) cibersexo solitario (principalmente visionado de pornografía), 3) cibersexo social (uso de chats con fines sexuales), 4) gasto económico online (inversión económica durante la práctica del cibersexo) y 5) percepción de gravedad (en qué medida el paciente considera grave su propio comportamiento sexual online). Ballester y cols. (2010)

informan de una fiabilidad de ,88 para la escala general y de entre ,49 y ,81 para las subescalas. La estabilidad temporal ( $r$ test-retest) fue de ,82. para la escala general y de entre , 43 y ,86 para las subescalas.

\section{Procedimiento}

Tal como recomiendan los protocolos de traducción de cuestionarios, la traducción del IH del inglés al español se realizó mediante metodología grupal. Cuatro miembros del equipo de investigación realizaron traducciones independientes del instrumento que después se pusieron en común con el fin de consensuar una versión preliminar. Esta versión fue administrada a 30 jóvenes para comprobar que resultaba comprensible y no inducía a interpretaciones erróneas. Tras realizar las mejoras que sugirieron, se dispuso la versión final (anexo 1).

La traducción del IH junto con el resto de instrumentos se administraron en mesas informativas habilitadas en las puertas de entrada a las distintas facultades de la Universitat Jaume I. Los estudiantes que accedían a participar completaban el consentimiento informado y los instrumentos de evaluación de forma anónima y en lápiz y papel. A un pequeño porcentaje se les ofreció la oportunidad de colaborar en una segunda fase. Los que aceptaron, acudieron a un laboratorio donde completaron, bajo supervisión, los instrumentos empleados para el cálculo de la validez convergente. 200 estudiantes accedieron a participar en esta segunda fase. 


\section{Análisis de datos}

En primer lugar, se exploró la estructura factorial del IH mediante un análisis factorial exploratorio (AFE) realizado con el software estadístico SPSS 21.0. La extracción de factores se realizó a través del método de componentes principales y se aplicó una rotación oblicua (Oblimin). Sobre el modelo factorial resultante, se extrajeron diversos estadísticos descriptivos (rango, media, desviación típica y asimetría) y se calcularon tres índices de fiabilidad ( $\alpha$ de Cronbach, correlación ítem-escala y correlación ítem-test). También se obtuvo la validez de la estructura factorial correlacionando ( $r$ de Pearson) la puntuación general y las subescalas del $\mathrm{IH}$ con medidas teóricamente relacionadas. Finalmente, se comprobó el ajuste de dos modelos factoriales a través de un análisis factorial confirmatorio (AFC) realizado con el software EQS 6.2. El ajuste del modelo se evaluó a través de los siguientes índices de ajuste: el chi cuadrado de Satorra-Bentler $\left(X^{2}\right)$, el chi cuadrado relativo ( $X^{2} / G$.L.), la significación general del modelo ( $p$ ), la raíz cuadrada media del error de aproximación (RMSEA) y los índices de ajuste comparativo (CFI) e incremental (IFI). Se consideró que el ajuste del modelo era correcto cuando el $X^{2}$ resultaba no significativo ( $\left.p>, 05\right)$, el $X^{2} / G$.L. se situaba entre 1 y 2 , el CFI y el IFI eran $\geq, 95$ y el RMSEA $\leq, 05$ (Bagozzi y Yi, 2011). Tomando criterios laxos, valores entre 2 y 3 para el X²/G.L., $\geq .90$ para el CFI y el IFI y $\leq, 08$ para el RMSEA se consideraron aceptables.

\section{Resultados}

Para comprobar la viabilidad de la aplicación de un AFE al IH, se calculó en primer lugar el índice de Kaiser-Meyer-Olkin $(\mathrm{KMO}=0,936)$ y la prueba de esfericidad de Bartlett $\left(X^{2}(171)=5033,13 ; p<0,001\right)$. Los resultados en ambas pruebas indicaban que el cuestionario admitía una solución factorial. A través del análisis de la matriz de correlaciones antimagen, se comprobó además la adecuación de los 19 ítems para su análisis mediante AFE (todas las correlaciones antimagen $>, 92$ ).

Tabla 1

Pesos factoriales, estadísticos descriptivos y coeficientes de fiabilidad para los ítems, factores y puntuación total del IH

\begin{tabular}{|c|c|c|c|c|c|c|c|c|c|c|}
\hline \multirow{2}{*}{\multicolumn{2}{|c|}{ Ítems }} & \multicolumn{3}{|c|}{ Pesos factoriales } & \multirow[t]{2}{*}{ Rango } & \multirow[t]{2}{*}{$M(D T)$} & \multirow[t]{2}{*}{ Asimetría } & \multicolumn{3}{|c|}{$\begin{array}{l}\text { Coeficientes } \\
\text { de fiabilidad }\end{array}$} \\
\hline & & Factor 1 & Factor 2 & Factor 3 & & & & $\alpha$ & $\mathrm{I}-\mathrm{S} r$ & $\mathrm{I}-\mathrm{T} r$ \\
\hline \multirow{7}{*}{ 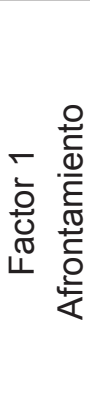 } & Ítem 1 & ,802 & & & $1-5$ & $2,20(1,08)$ & 0,59 & NA & ,787 & ,668 \\
\hline & Ítem 3 & ,687 & & & $1-5$ & $1,83(1,11)$ & 1,05 & NA & ,701 & ,643 \\
\hline & Ítem 6 & ,776 & & & $1-5$ & $1,64(0,97)$ & 1,58 & NA & ,770 & ,685 \\
\hline & Ítem 8 & ,780 & & & $1-5$ & $2,06(1,14)$ & 0,84 & NA & ,797 & ,726 \\
\hline & Ítem 13 & ,763 & & & $1-5$ & $2,32(1,17)$ & 0,55 & NA & ,761 & ,647, \\
\hline & Ítem 16 & ,759 & & & $1-5$ & $1,60(0,91)$ & 1,52 & NA & ,778 & ,725 \\
\hline & Ítem 18 & ,704 & & & $1-5$ & $1,52(0,87)$ & 1,89 & NA & ,749 & ,716 \\
\hline
\end{tabular}




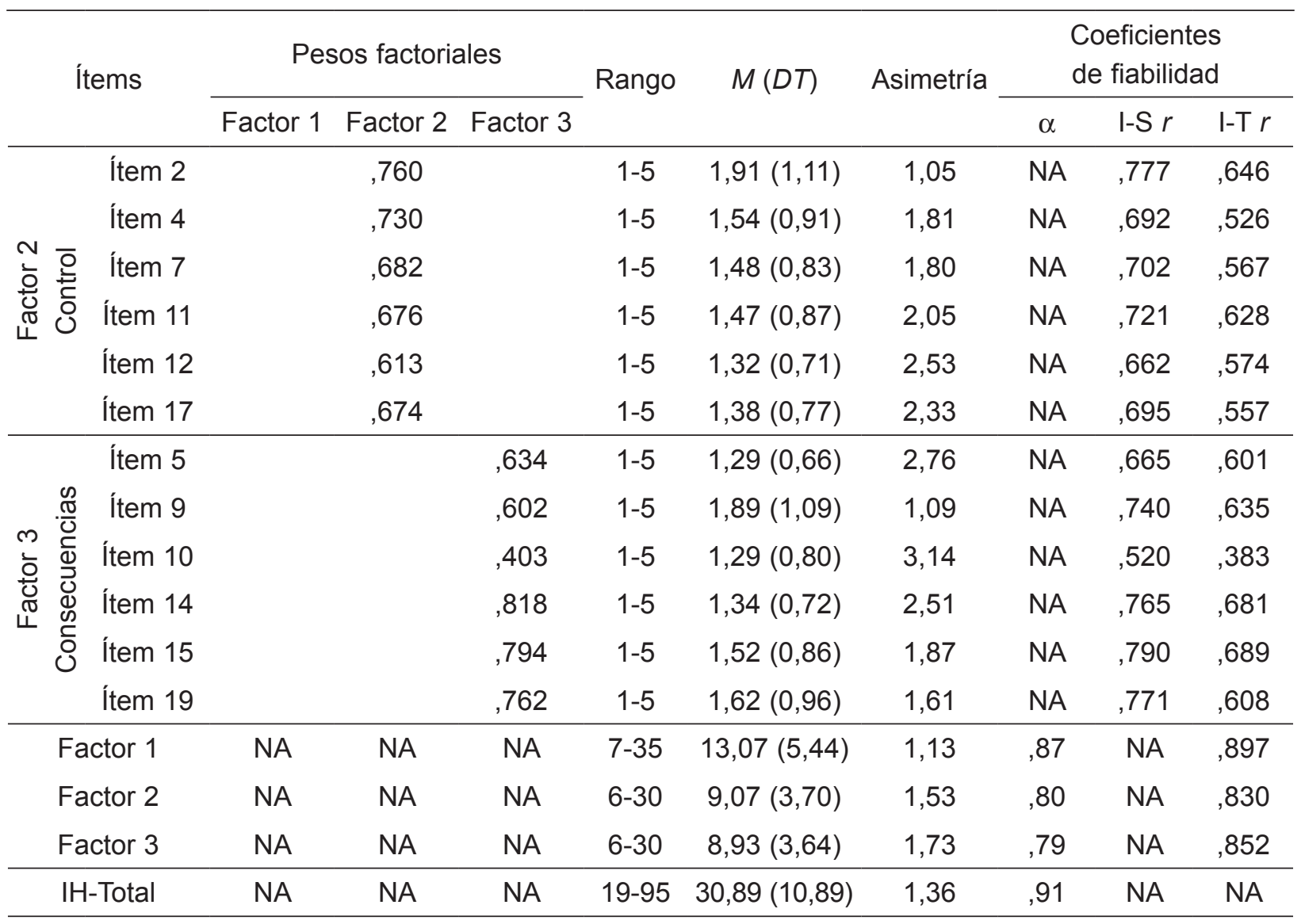

${ }^{*} p<0,05 ;{ }^{* *} p<0,01 ;{ }^{* * *} p<0,001$

Nota: NA = no aplicable; I-S $r=$ correlación ítem-escala corregida; I-T $r=$ correlación ítem-test corregida.

Para evitar generar subescalas con poco peso explicativo, se limitó la extracción de factores a autovalores (eigenvalues) superiores a 1,5. Con este criterio, la solución factorial derivada del AFE reveló una estructura de 3 factores que explicaban el 55,5\% de la varianza del cuestionario (tabla 1). El primer factor explicaba un 40,99 \% de la varianza del cuestionario. Este factor agrupó 7 ítems relacionados con el uso del sexo como regulador de estados emocionales disfóricos (por ejemplo, "Cuando me siento inquieto, recurro al sexo para calmarme») y recibió el nombre de afrontamiento. El segundo factor explicaba el 8,31\% de la varianza y comprendía 6 ítems relacionados con la incapacidad para controlar el comportamiento sexual (por ejemplo, «Participo en actividades sexuales sabiendo que más tarde me arrepentiré»), de modo que recibió el nombre de control. El tercer factor agrupó los 6 ítems restantes y permitía explicar el 6,24 \% de la varianza. Los ítems en este factor hacían referencia a las consecuencias del comportamiento sexual en diferentes ámbitos (por ejemplo, «Mis actividades sexuales interfieren en algunos aspectos de mi vida»), de modo que fue bautizado como consecuencias.

Como se aprecia en la tabla 1 , muchos de los ítems del IH presentaban una asimetría positiva moderada-alta (13 de los 19 ítems con valores de asimetría $>1,5$ ), sugiriendo una tendencia a responder sobre todo con los valores más bajos del rango de respuestas (entre nunca y pocas veces). La asimetría se suaviza en las puntuaciones de los 3 factores y sobre todo en la puntuación total del cuestionario (asimetría de 1,36), con valores esperables para una escala de screening psicopatológico. Teniendo en cuenta que el rango de puntuaciones entre escalas 
no es equivalente, la puntuación media mayor se da en el factor 1 (rango = 7-35; $M=13,07$ ), seguido del factor 2 (rango $=6-30 ; M=9,07$ ) y el 3 (rango $=6-30 ; M=8,93$ ).

Por lo que se refiere a la fiabilidad, el alpha de Cronbach de todos los factores se situó por encima de ,79 (tabla 1). Especialmente reseñable sería la fiabilidad de la escala general $(\alpha=, 91)$, que superaría holgadamente el criterio de Henson (2001) de ,80 para considerar una escala fiable. Las correlaciones ítem-escala e ítem-test fueron aceptables, con valores entre , $520-, 797$ y ,383-,726 respectivamente.

Tabla 2

Correlaciones (Pearson) entre el IH y otras medidas

\begin{tabular}{|c|c|c|c|c|c|}
\hline & & \multicolumn{4}{|c|}{ Inventario de hipersexualidad $(\mathrm{IH})$} \\
\hline & & IH-Total & Factor 1 & Factor 2 & Factor 3. \\
\hline \multirow{3}{*}{$\begin{array}{l}\text { Escala de compulsividad } \\
\text { sexual (scs) } \\
\qquad(n=600)\end{array}$} & Scs-Total &, $712^{* * *}$ &, $569^{* * *}$ & $628^{* * *}$ &, $681^{* * *}$ \\
\hline & Scs-Control &, $672^{\star \star *}$ &, $559^{* * *}$ &, $571^{* * *}$ &, $628^{* * *}$ \\
\hline & Scs-Interferencia &, $589^{* * *}$ &, $441^{* * *}$ &, $554^{\star * *}$ &, $587^{* * *}$ \\
\hline \multirow{5}{*}{$\begin{array}{l}\text { Cuestionario de adicción } \\
\text { al sexo (SAST) } \\
(n=600)\end{array}$} & SAST-Total &, $687^{* * *}$ &, $533^{* * *}$ &, $639^{* * *}$ &, $630^{* * *}$ \\
\hline & SAST-Falta de control &, $523^{* * *}$ &, $362^{* * *}$ &, $594^{* * *}$ &, $435^{* * *}$ \\
\hline & SAST-Síntomas &, $690^{* * *}$ &, $566^{* * *}$ &, $564^{\star * \star}$ &, $690^{* * *}$ \\
\hline & SAST-Interferencia familiar &, $252^{* * *}$ &, $154^{* * *}$ &, $284^{* * *}$ &, $281^{* * *}$ \\
\hline & SAST-Ocultación &, $309^{* * *}$ &, $271^{* * *}$ &, $280^{* * *}$ &, $243^{* * *}$ \\
\hline $\begin{array}{l}\text { Escala de búsqueda de } \\
\text { sensaciones sexuales } \\
\qquad(\text { BSs }) \\
(n=200)\end{array}$ & Bss-Total &, $473^{* * *}$ &, $420^{* * *}$ &, $334^{\star * *}$ &, $410^{* * *}$ \\
\hline \multirow{6}{*}{$\begin{array}{l}\text { Cuestionario de adicción } \\
\text { al cibersexo (ISST) } \\
(n=200)\end{array}$} & ISST-Total &, $501^{\star * *}$ &, $388^{* * *}$ &, $482^{* * *}$ &, $407^{* * *}$ \\
\hline & $\begin{array}{l}\text { ISST-Compulsividad sexual } \\
\text { online }\end{array}$ &, $402^{* * *}$ &, $338^{* * *}$ &, $381^{* * *}$ &, $266^{* * *}$ \\
\hline & ISST-Cibersexo solitario &, $363^{* * *}$ &, $310^{\star * *}$ &, $295^{\star \star *}$ &, $335^{\star * *}$ \\
\hline & ISsT-Cibersexo social &, $450^{\star * *}$ &, $313^{* * *}$ &, $485^{\star * *}$ &, $347^{* * *}$ \\
\hline & ISST-Gasto económico &, $191^{* *}$ & ,096 &, $199^{* * *}$ &, $199^{* * *}$ \\
\hline & $\begin{array}{l}\text { ISsT-Percepción de grave- } \\
\text { dad }\end{array}$ &, $169^{*}$ & ,089 &, $195^{\star \star *}$ & ,137 \\
\hline
\end{tabular}

${ }^{*} p<0,05 ;{ }^{* *} p<0,01 ;{ }^{* * *} p<0,001$

Para el análisis de la validez del IH, esta medida se correlacionó con otros instrumentos empleados en la medición del mismo constructo o dimensiones afines (tabla 2). Así, se encontró que las correlaciones entre las escalas y subescalas del IH y de los cuestionarios SCS y SAST fueron en todos los casos positivas, altas y significativas a nivel $p<, 001$. De las escalas del $\mathrm{IH}$, la que se 
relacionó con mayor intensidad con los otros dos cuestionarios de screening fue la puntuación total (correlaciones entre ,252-,690 con el SAST y de entre ,589-,712 con el SCS). Las correlaciones entre el IH y el ISST -en particular con la puntuación total- fueron también altas y significativas excepto para las subescalas de gasto económico y de percepción de gravedad, donde la correlación fue signifiativa pero de magnitud menor. Así mismo, la escala BSs correlacionó de forma significativa con la puntuación total y las 3 subescalas del IH ( $r$ entre ,334 y ,473).

Para contrastar empíricamente la estructura factorial del IH, se realizó un AFC por medio del programa de ecuaciones estructurales EQS 6.2 y aplicando el método de ML Robusto (método que permite obtener estadísticos que corrigen el efecto de violación del principio de normalidad). A través de esta metodología se contrastaron dos modelos: el primero (M1) replicaba la estructura factorial obtenida durante el AFE (tres factores de primer orden correlacionados) mientras que el segundo (M2) proponía que los tres factores de primer orden se agrupaban bajo uno de segundo orden que explicaba la varianza compartida. En la tabla 3 figuran los índices de bondad de ajuste de ambos modelos.

Tabla 3

Índices de bondad de ajuste para los modelos confirmatorios 1 y 2

\begin{tabular}{cccccccc}
\hline & $\chi^{2}$ & G.L. & $p$ & $x^{2} / G . L$. & RMSEA & CFI & IFI \\
\hline M1 & 331,82 & 149 & $<, 001$ & 2,22 &, 045 &, 91 &, 91 \\
M2 & 239,46 & 145 & $<, 001$ & 1,65 &, 03 &, 71 &, 73 \\
\hline
\end{tabular}

Nota: $\mathrm{x}^{2}=\mathrm{x}^{2}$ de Satorra-Bentler; $\mathrm{x}^{2} / \mathrm{G}$.L. $=\mathrm{x}^{2}$ relativo; GFI = estadístico de bondad de ajuste; $\mathrm{AGFI}=$ estadístico de bondad de ajuste corregido; $\mathrm{RMSEA}=$ raíz cuadrada media del error de aproximación; $\mathrm{CFI}=$ índice de ajuste comparativo; $I F I=$ índice de ajuste incremental.

Como se desprende de la tabla 3, el modelo cuyos índices de ajuste resultan más satisfactorios es el segundo (M2). En este modelo, el valor de significación del $x^{2}$ de Satorra-Bentler ( $x^{2}$ corregido para muestras que no siguen el supuesto de normalidad) no superaría el criterio de ,05 para considerarlo satisfactorio. Sin embargo, se ha demostrado que este estadístico se encuentra altamente condicionado por el tamaño muestral, que en nuestro caso supera en mucho el mínimo exigido para estos análisis. Si prestamos atención a otros índices menos sensibles al tamaño muestral, el valor del $X^{2}$ relativo ( $X^{2} / G . L$.) para el modelo 2 (M2) es de 1,65, considerándose un ajuste aceptable valores por debajo del 2 (Bagozzi y Yi, 2011). Otro estadístico que da buena cuenta del ajuste de M2 es el RMSE, que se sitúa por debajo del valor de 0,05 que exigen los criterios más estrictos para considerar un modelo parsimonioso. En contraposición, los estadísticos CFI e IFI muestran un mejor ajuste para el M1 que para el M2, si bien estos se consideran secundarios a los ya comentados.

\section{Discusión y conclusiones}

En este trabajo se presentan los resultados de la adaptación y validación al castellano de uno de los principales instrumentos empleados para el diagnóstico de la hipersexualidad: 
el inventario de hipersexualidad. Además, este trabajo se ha realizado con jóvenes de entre 18 y 27 años, reconociendo así la importancia de este periodo en la manifestación de los primeros síntomas de hipersexualidad y la aparición de las primeras consecuencias negativas asociadas (Reid, Garos y Fong, 2012). En este sentido, el IH no solo se dibuja como un instrumento eficaz para la evaluación de este cuadro clínico en jóvenes, sino que además resulta superior a otros empleados con tanta o más frecuencia con fines clínicos y empíricos.

Con respecto a la estructura factorial del cuestionario, se ha demostrado que los 19 ítems del IH se dividen en 3 escalas que se agrupan a su vez bajo un único factor de segundo orden al que hemos llamado hipersexualidad. Este factor de orden superior sería el equivalente a la descripción diagnóstica general del cuadro clínico de hipersexualidad, mientras que las 3 subescalas representan los principales grupos de síntomas de esta patología. De estas tres escalas, la que ha mostrado mayor peso en el cuestionario es la de afrontamiento, donde se agrupan ítems cuyo contenido explora el uso del sexo como regulador emocional. Si bien es cierto que muchos de estos pacientes se caracterizan por emplear el sexo como válvula de escape ante estados de ánimo disfóricos (ansiedad, depresión, ira, etc.) (Reid, Carpenter y cols., 2012), el peso de estos síntomas suele ser menor al de otros como por ejemplo los problemas en el control de impulsos sexuales. Así sería por ejemplo en la validación en castellano del cuestionario de adicción al sexo, donde los autores encontraron un mayor peso de los síntomas de control de impulsos sobre los de regulación emocional (Castro-calvo y cols., 2016). El siguiente factor en importancia sería ahora sí el de control, que comprendía los ítems que hacen referencia a la capacidad para manejar de forma voluntaria la conducta sexual. Este factor permitiría, por tanto, discriminar entre aquellos que simplemente muestran un deseo sexual un tanto más elevado y los que realmente padecen de una adicción sexual (que combinarían un deseo sexual desmedido y una incapacidad patológica para su control) (Walters, Knight y Långström, 2011). Finalmente, el factor consecuencias explora la interferencia generada por el cuadro clínico en diferentes ámbitos (sobre todo a nivel personal) y que representa un criterio básico en el diagnóstico de cualquier patología mental.

Un aspecto que refuerza la validez de la estructura factorial obtenida es que la distribución de ítems por cada una de las tres escalas coincide exactamente con la obtenida por los autores de la versión original del cuestionario (Reid y cols., 2011). Estos investigadores validaron esta escala en población clínica, de modo que la obtención de una estructura factorial equivalente en población general no solo supone una prueba a favor de la concepción dimensional -y no categórica- de esta problemática (Walters y cols., 2011), sino que, además, apoya su empleo en otros contextos socioculturales más allá de aquellos para los que el cuestionario fue diseñado. Finalmente, tanto la fiabilidad del $\mathrm{IH}$-en particular la de la escala total $(\alpha=, 91)$ - como su validez en la evaluación clínica de la hipersexualidad quedan sobradamente demostrados en este trabajo.

\section{Referencias bibliográficas}

Bagozzi, R. P. y Yi, Y. (2011). Specification, evaluation, and interpretation of structural equation models. Journal of the Academy of Marketing Science, 40, 8-34.

Ballester-Arnal, R., Gil-Llario, M. D., Gómez-Martínez, S. y Gil-Julià, B. (2010). Psychometric properties of an instrument for assessing cyber-sex addiction. Psicothema, 22, 10481053.

Ballester-Arnal, R., Gómez-Martínez, S., Gil-Llario, M. D. y Salmerón-Sánchez, P. (2013). Sexual compulsivity scale: adaptation and validation in the spanish population. Journal of Sex \& Marital Therapy, 39, 526-540. 
Castro-Calvo, J., Ballester-Arnal, R. y Gil-Llario, M. D. (2015). Compulsividad Sexual: Convergencia y prevalencia entre medidas. Agora de Salut, 2, 205-213.

Castro-Calvo, J., Ballester-Arnal, R., Salmerón-Sánchez, P. y Gil-llario, M. D. (2016). Traducción y validación preliminar del cuestionario de adicción al sexo en jóvenes. International Journal of Developmental and Educational Psychology, 2, 319-328.

Hagedorn, W. B. (2009). Sexual addiction counseling Competencies: Empirically-based tools for preparing clinicians to recognize, assess, and treat sexual addiction. Sexual Addiction \& Compulsivity, 16, 190-209.

Kafka, M. P. (2010). Hypersexual disorder: a proposed diagnosis for DSM-V. Archives of Sexual Behavior, 39, 377-400.

Kalichman, S. C., y Rompa, D. (1995). Sexual sensation seeking and Sexual Compulsivity Scales: reliability, validity, and predicting HIV risk behavior. Journal of Personality Assessment, 65, 586-601.

Reid, R. C., Carpenter, B. N., Hook, J. N., Garos, S., Manning, J. C., Gilliland, R. y cols. (2012). Report of findings in a DSM-5 field trial for hypersexual disorder. The Journal of Sexual Medicine, 9, 2868-2877.

Reid, R. C., Garos, S. y Carpenter, B. N. (2011). Reliability, validity, and psychometric development of the Hypersexual Behavior Inventory in an outpatient sample of men. Sexual Addiction \& Compulsivity, 18, 30-51.

Reid, R. C., Garos, S. y Fong, T. (2012). Psychometric development of the hypersexual behavior consequences scale. Journal of Behavioral Addictions, 1, 115-122.

Rettenberger, M., Klein, V. y Briken, P. (2015). The relationship between hypersexual behavior, sexual excitation, sexual inhibition, and personality traits. Archives of Sexual Behavior, First Publ, 1-15.

Wines, D. (1997). Exploring the applicability of criteria for substance dependence to sexual addiction. Sexual Addiction \& Compulsivity, 4, 195-220.

Winters, J., Christoff, K. y Gorzalka, B. B. (2010). Dysregulated sexuality and high sexual desire: distinct constructs? Archives of Sexual Behavior, 39, 1029-1043.

Womack, S., Hook, J., Ramos, M., Davis, D. E. y Penberthy, J. K. (2013). Measuring hypersexual behavior. Sexual Addiction \& Compulsivity, 20, 65-78. 
Anexo 1

Inventario de hipersexualidad (traducido y adaptado por Salusex-Unisexsida)

A la hora de completar este cuestionario, recuerda que entendemos como sexo, cualquier actividad o conducta que estimula o excita a una persona con la intención de producir un orgasmo o placer sexual (por ejemplo, masturbación o sexo en solitario, uso de pornografía, relaciones sexuales con una pareja, sexo oral, sexo anal, etc.). Las conductas sexuales pueden o no implicar a una pareja.

\begin{tabular}{|c|c|c|c|c|c|}
\hline Ítem & Nunca & $\begin{array}{l}\text { Pocas } \\
\text { veces }\end{array}$ & $\begin{array}{c}\text { Algunas } \\
\text { veces }\end{array}$ & $\begin{array}{l}\text { Bastantes } \\
\text { veces }\end{array}$ & $\begin{array}{l}\text { Muchas } \\
\text { veces }\end{array}$ \\
\hline $\begin{array}{l}\text { 1. Uso el sexo para olvidarme de las preocupacio- } \\
\text { nes cotidianas. }\end{array}$ & 1 & 2 & 3 & 4 & 5 \\
\hline $\begin{array}{l}\text { 2. Aunque me prometo no repetir ciertas conductas } \\
\text { sexuales, las hago nuevamente. }\end{array}$ & 1 & 2 & 3 & 4 & 5 \\
\hline $\begin{array}{l}\text { 3. Hacer cualquier cosa relacionada con el sexo me } \\
\text { ayuda a sentirme menos solo/a. }\end{array}$ & 1 & 2 & 3 & 4 & 5 \\
\hline $\begin{array}{l}\text { 4. Participo en actividades sexuales sabiendo que } \\
\text { luego me arrepentiré. }\end{array}$ & 1 & 2 & 3 & 4 & 5 \\
\hline $\begin{array}{l}\text { 5. Sacrifico cosas que realmente quiero en mi vida } \\
\text { por el sexo. }\end{array}$ & 1 & 2 & 3 & 4 & 5 \\
\hline $\begin{array}{l}\text { 6. Recurro al sexo cuando experimento sentimientos } \\
\text { desagradables. }\end{array}$ & 1 & 2 & 3 & 4 & 5 \\
\hline $\begin{array}{l}\text { 7. Fracaso en mis intentos por cambiar mi conducta } \\
\text { sexual. }\end{array}$ & 1 & 2 & 3 & 4 & 5 \\
\hline $\begin{array}{l}\text { 8. Cuando me siento inquieto, recurro al sexo para } \\
\text { calmarme. }\end{array}$ & 1 & 2 & 3 & 4 & 5 \\
\hline $\begin{array}{l}\text { 9. Mis pensamientos y fantasías sexuales me dis- } \\
\text { traen de cumplir tareas. }\end{array}$ & 1 & 2 & 3 & 4 & 5 \\
\hline $\begin{array}{l}\text { 10. Practico actividades sexuales que están en contra } \\
\text { de mis valores y creencias. }\end{array}$ & 1 & 2 & 3 & 4 & 5 \\
\hline $\begin{array}{l}\text { 11. Aunque mi conducta sexual sea irresponsable, } \\
\text { tengo dificultades para detenerla. }\end{array}$ & 1 & 2 & 3 & 4 & 5 \\
\hline $\begin{array}{l}\text { 12. Siento que mi conducta sexual me lleva en una } \\
\text { dirección a la que no quiero ir. }\end{array}$ & 1 & 2 & 3 & 4 & 5 \\
\hline $\begin{array}{l}\text { 13. Hacer cualquier cosa relacionada con el sexo me } \\
\text { ayuda a manejar mi estrés. }\end{array}$ & 1 & 2 & 3 & 4 & 5 \\
\hline 14. Mi conducta sexual controla mi vida. & 1 & 2 & 3 & 4 & 5 \\
\hline $\begin{array}{l}\text { 15. Siento que mis ansias y deseos sexuales son } \\
\text { más fuertes que mi autodisciplina. }\end{array}$ & 1 & 2 & 3 & 4 & 5 \\
\hline
\end{tabular}




\begin{tabular}{|l|c|c|c|c|c|}
\hline \multicolumn{1}{|c|}{ Ítem } & Nunca & $\begin{array}{c}\text { Pocas } \\
\text { veces }\end{array}$ & $\begin{array}{c}\text { Algunas } \\
\text { veces }\end{array}$ & $\begin{array}{c}\text { Bastantes } \\
\text { veces }\end{array}$ & $\begin{array}{c}\text { Muchas } \\
\text { veces }\end{array}$ \\
\hline $\begin{array}{l}\text { 16. El sexo es para mí una forma de manejar el ma- } \\
\text { lestar emocional que siento. }\end{array}$ & 1 & 2 & 3 & 4 & 5 \\
$\begin{array}{l}\text { 17. Sexualmente, me comporto de formas que creo } \\
\text { que están mal. }\end{array}$ & 1 & 2 & 3 & 4 & 5 \\
$\begin{array}{l}\text { 18. Uso el sexo como una forma de ayudarme a } \\
\text { afrontar mis problemas. }\end{array}$ & 1 & 2 & 3 & 4 & 5 \\
$\begin{array}{l}\text { 19. Mis actividades sexuales interfieren en algunos } \\
\text { aspectos de mi vida. }\end{array}$ & 1 & 2 & 3 & 4 & 5 \\
\hline
\end{tabular}

